\title{
Scatter Matrix Concordance: A Diagnostic for Regressions on Subsets of Data
}

\author{
Michael J. Kane ${ }^{1}$, Bryan Lewis ${ }^{2}$, Sekhar Tatikonda ${ }^{3}$, and Simon \\ Urbanek $^{4}$ \\ ${ }^{1}$ Yale University and Phronesis LLC, New Haven CT, USA \\ ${ }^{2}$ Paradigm4, Waltham MA, USA \\ ${ }^{3}$ Yale University, New Haven CT, USA \\ ${ }^{4}$ AT\&T Labs Research, New York NY, USA
}

July 14, 2015

\begin{abstract}
Linear regression models depend directly on the design matrix and its properties. Techniques that efficiently estimate model coefficients by partitioning rows of the design matrix are increasingly popular for large-scale problems because they fit well with modern parallel computing architectures. We propose a simple measure of concordance between a design matrix and a subset of its rows that estimates how well a subset captures the variance-covariance structure of a larger data set. We illustrate the use of this measure in a heuristic method for selecting row partition sizes that balance statistical and computational efficiency goals in real-world problems.
\end{abstract}

\section{Introduction}

A common procedure in supervised learning problems when the number of rows of data is large and far exceeds the number of columns is to partition the rows, fit models on individual partitions, and combine them by averaging or other aggregation into a single model. This computational approach is referred to as "Divide and Recombine" (D\&R) in Guha et al. (2012) and has gained wide use in part because it can be described as a single MapReduce (Dean and Ghemawat, 2008) step and is easily implemented in software frameworks like Hadoop (Apache Software Foundation, 2014) and Spark (Zaharia et al., 2010).

Partitions are constructed either by conditioning-variable division or replicate division. The former adds samples to a partition based on one or more of the variables in the data. For categorical variables this generally means one partition per level and is equivalent to a variable interaction between the categorical variable being conditioned 
on all other regressors in the model. For continuous variables this means one partition per range of values. Alternatively, data can be partitioned along multiple conditioning variables. Replicate division creates partitions using random sampling without replacement.

Guha et al. (2012) provide conditions such that a model constructed by averaging coefficients from ordinary least squares models along replicate division data partitions converges asymptotically to the single ordinary least squares model fitted to all of the data, called the reference model. We constrain our attention to D\&R under replicate division to create a single, averaged model.

Note that the D\&R method is related to boosting since in both cases an ensemble of models is created by sampling from the original data set. There are differences, however. First, when boosting samples with replacement the D\&R averaged model samples without replacement. Second, where the result of training a boosted model is an ensemble of individual learners the result of training a D\&R model is a single model where parameters have been averaged together.

D\&R describes a general computational approach to large scale regression and classification requiring only a single pass through the data set. This gives D\&R a computational advantage over those models which require multiple passes through the data. Matloff (2014) extends both the statistical results by providing a broader class estimators under which D\&R converges as well as the complexity analysis by showing that the computational speed up can be greater than the number of parallel processes applied to each division. Kleiner et al. (2011) also developed the statistical theory further by identifying a general class of models that converge under the random partition assumption as well as showing that bootstrap models can be created on each partition and the ensemble of models over all partitions converges asymptotically to a single bootstrap model over the entire data set.

There are at least two practical issues to consider when implementing the D\&R approach to model fitting with replicate division on distributed data. First, partitions should consist of random sets of rows to ensure that the aggregate model converges to its reference. This is important in real-world, finite-sized problems to avoid artifacts related to ordering in the data, for example from the data collection process. If samples are arranged in partitions, without randomization, then individual partitions may capture information that is drastically different from the other partitions or the total population. As a result, an aggregate model can perform poorly when compared to its reference.

The second challenge is in deciding the number of samples per block. The convergence results show that the ensemble of models converges to the reference model asymptotically in the number of random samples in the partitions. This implies that, to minimize the difference between the ensemble and the reference, block sizes should be as large as possible. However, the amount of speed-up achieved is generally directly proportional to the number of partitions (assuming each block is allocated its own process). Therefore, there is a trade-off between the statistical consistency of a model created using the D\&R approach (with respect to its reference) and the parallelism that can be achieved when fitting the model.

The regression techniques presented work on subsets of disjoint rows and, with this observation, a natural subsequent question is how well do these individual subsets 
represent the data set as a whole? This is a relevant question since if we have a small representative subset then we may not need all of the data. We may be able to fit a model more efficiently by only using the representative subset. If we find that some subsets differ drastically from the rest of the data set we may need to investigate these subsets further. They may indicate different underlying relationships that can be conditioned upon or they may indicate data integrity issues. In either case they motivate further investigation.

This paper explores the trade-off between statistical consistency and computational efficiency by introducing a statistic that estimates the concordance between a subset and the entire data set based on their respective variance-covariance structures. We provide reproducible experiments that illustrate our concept of concordance and its use along with benchmark results. A final section is devoted to discussion and future directions.

\section{Motivating Case: Least Squares Regression}

Consider the following example linear model and corresponding least squares problem:

$$
Y=\mathbf{X} \beta+\varepsilon
$$

where $Y, \varepsilon \in \mathcal{R}^{n}$, and $\beta \in \mathcal{R}^{d}, n \geq d$; each element of $\varepsilon$ is an i.i.d. random variable with mean zero; and $\mathbf{X}$ is a matrix in $\mathcal{R}^{n \times d}$ with full column rank. The ordinary least squares problem is posed as $\widehat{\beta}=\operatorname{argmin}_{\beta}\|\mathbf{X} \beta-Y\|^{2}$ and has the closed form analytic solution defined by the normal equations:

$$
\widehat{\beta}=\left(\mathbf{X}^{T} \mathbf{X}\right)^{-1} \mathbf{X}^{T} Y .
$$

We remark that computed solutions rarely use Equation 2 directly but rather use QR or SVD decompositions of $\mathbf{X}$ for numerical stability. Equation 2 remains important for analysis purposes. Consider the row-wise partitioning of Equation 1 .

$$
\left[\begin{array}{c}
Y_{1} \\
Y_{2} \\
\vdots \\
Y_{r}
\end{array}\right]=\left[\begin{array}{c}
\mathbf{X}_{1} \\
\mathbf{X}_{2} \\
\vdots \\
\mathbf{X}_{r}
\end{array}\right] \beta+\left[\begin{array}{c}
\varepsilon_{1} \\
\varepsilon_{2} \\
\vdots \\
\varepsilon_{r}
\end{array}\right]
$$

where $Y_{1}, Y_{2}, \ldots, Y_{r}, \mathbf{X}_{1}, \mathbf{X}_{2}, \ldots, \mathbf{X}_{r}$ and $\varepsilon_{1}, \varepsilon_{2}, \ldots, \varepsilon_{r}$ are data partitions such that each block is composed of subsets of rows of a data set, blocks are disjoint, and the aggregate of all blocks is the original data set. Without loss of generality we assume that $n / r=c$ is an integer so that the number of samples in each submatrix is the same. The blocks may, for example, be located in files across a network of computers for distributed computation.

When each of the $\mathbf{X}_{i}$ 's represent a random partition of $\mathbf{X}$ then the estimate for least squares regression coefficients using D\&R averages the block-wise estimates of the slope coefficients $\tilde{\beta}_{i}$,

$$
\tilde{\beta}_{i}=\left(\mathbf{X}_{i}^{T} \mathbf{X}_{i}\right)^{-1} \mathbf{X}_{i}^{T} Y_{i}, \quad \tilde{\beta}=\frac{1}{r} \sum_{i=1}^{r} \tilde{\beta}_{i} .
$$


Compare the D\&R approach to block-wise computation of the (full) least squares solution:

$$
\hat{\beta}=\left(\sum_{i=1}^{r} \mathbf{X}_{i}^{T} \mathbf{X}_{i}\right)^{-1} \sum_{i=1}^{r} \mathbf{X}_{i}^{T} Y_{i} .
$$

Both approaches are similarly easy to compute in parallel. The overall computational cost of both approaches is $O\left(d^{3}\right)$, although the $\mathrm{D} \& \mathrm{R}$ approach (Equation 3) has a larger constant term by a factor of $r$ compared to block-wise solution of the full problem (Equation 4).

Recall that the D\&R solution $\tilde{\beta}$ is an estimate of the true solution $\hat{\beta}$. It's reasonable to ask the why one would spend more computational effort to produce only an estimate of a solution, when a similarly easy direct solution method is available. Despite the apparent advantage of the direct block-wise solution method shown in Equation 4, the D\&R approach is potentially superior in two ways: lower network communication cost in parallel computing settings and better numerical stability. We outline each advantage below.

Lower network communication cost. Assume that the problem is distributed so that each data block $i=1,2, \ldots, r$ is located on a different computer in a network. The D\&R approach shown in Equation 3 averages $r$ sets of $d$ model coefficients to produce an averaged output model, for a total $r d$ numbers transmitted between computers over the network. Block-wise solution of the full problem outlined in 4, by comparison, sums $r$ partial $d \times d$ matrix products and $r$ vectors of length $d$, for a total of $r d^{2}+r d$ numbers to transmit over the network. The $d^{2}$ communication cost for the full solution is expensive when there are moderately large numbers of columns in the model matrix. For example with 8-byte double precision floating point numbers, $r=100$ and $d=$ 1000 , the full problem solution must transmit $800 \mathrm{MB}$ across the network. The D\&R approach only transmits $800 \mathrm{~KB}$ by comparison.

Better numerical stability. Although we routinely use the normal equations for analysis of least squares problems in exact arithmetic, their use computationally is not recommended because the matrix $\mathbf{X}^{T} \mathbf{X}$ is generally less well-conditioned (and never better conditioned) than $\mathbf{X}$. Instead, least squares problems are typically computed using either a QR or singular value decomposition of the model matrix $\mathbf{X}$. Unfortunately, parallel computation of a QR or SVD factorization for block row-distributed matrices is neither easy nor readily available to most high-level programming languages like $\mathrm{R}$ and Python, especially in MapReduce-like computing setting 1 . Note also that model matrices involving contrast variables derived from so-called factor variables are often sparse. SVD and QR decompositions destroy sparsity patterns and the resulting factorized model matrix may consume much more memory than the original. Distributed computation of the full problem is typically performed using the normal equations as shown in Equation 4 for these reasons. The D\&R approach, by contrast, is free to use numerically stable solution methods in each block. Moreover, since the blocks are relatively small, loss of a sparse model matrix representation in each block due to factorization is more tolerable.

\footnotetext{
${ }^{1}$ A notable exception is HPC systems using ScaLAPACK and MPI-see the R pbd package, for examplebut these are usually very specialized systems.
} 
We illustrate our point about numerical stability with a simple example.

$$
X=\left(\begin{array}{cc}
10^{9} & -1 \\
-1 & 10^{-5}
\end{array}\right), \quad \beta=\left(\begin{array}{l}
1 \\
1
\end{array}\right), \quad \text { and }, \quad y=X \beta .
$$

Note that $X$ is an ill-conditioned matrix, but not so ill-conditioned to prevent numericallystable techniques from working. This can be demonstrated using the $\mathrm{R}$ programming environment ( $R$ Core Team, 2014) to compare least square solutions of this example computed using a stable technique and using the normal equations.

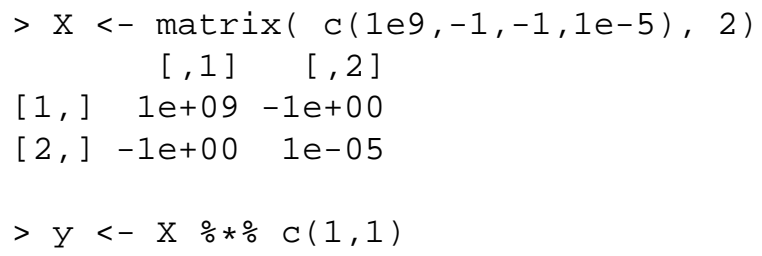

One stable least-squares solution approach gives us the expected solution:

$>$ qr.solve $(\mathrm{X}, \mathrm{y})$ $[, 1]$

$[1] \quad$,

$[2] \quad$,

Now with the normal equations. Note that in $\mathrm{R}$ we form the inverse of $X^{T} X$ as described in Equation 2

$>$ qr.solve $(t(x) \div * \% x) \div * \% t(x) \div * \% y$

Error in qr.solve : singular matrix ' $a$ ' in solve

An informative error. If we override the error, we get a bad result that is very different from what we expected:

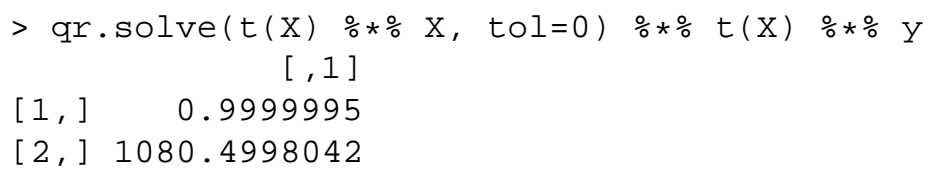

The example, although pathological by design, shows that using the normal equations directly to solve least squares problems can lead to failure or poor results. Even in better-conditioned problems, use of the normal equations will result in a loss of numerical accuracy in the solution. The D\&R method is free to employ numerically-stable techniques in each block.

The normal-equation approach does have the advantage of being easily updatable. data are received they can be added to a new block. If the block-coefficients are stored then updating the model is simply a matter of getting the estimate for the new block and once again averaging over all of the coefficient estimates. The removal of data or the updating of an existing block can be handled similarly. The D\&R method is only updatable when the incoming data are guaranteed to be distributed at random, that is there is no local correlation structure that exists only a new block. The effect of non-randomness is explored further in Section 6.2 


\section{Common-basis Concordance}

The previous section shows the trade-offs and characteristics of estimating the slope coefficients in a ordinary linear regression using the normal equations along with D\&R. In both cases the slope coefficients are a function of the variance-covariance structure among regression and regressor variables and it seems reasonable that fitting subsets a subset of data could give similar results and require less computational complexity. This section introduces concordance as a statistic for capturing how well a subset of data represents the whole. For this paper, a data set is "representative" of a larger data set if the correlation structure of the two data sets are similar and we are most interested in the case where one data set is a small subset of the rows of a larger data set. In Section 7 other potential applications and directions are proposed.

Let $\mathbf{A}$ and $\mathbf{B}$ be $n \times d$ and $m \times d$ matrices respectively The previous section is meant to motivate with $m, n \geq d$; let $\mathbf{A}^{T} \mathbf{A}$ and $\mathbf{B}^{T} \mathbf{B}$ have eigenvalue decompositions $\mathbf{V}^{T} \lambda_{A} \mathbf{V}$ and $\mathbf{V}^{T} \lambda_{B} \mathbf{V}, \mathbf{V}^{T} \mathbf{V}=\mathbf{I}$, respectively; and assume $\left(\mathbf{A}^{T} \mathbf{A}\right)^{-1}$ and $\left(\mathbf{B}^{T} \mathbf{B}\right)^{-1}$ exist. The concordance of these two matrices is defined as

$$
S(\mathbf{A}, \mathbf{B})=\frac{n}{d m}\left\|\mathbf{A} \mathbf{B}^{\dagger}\right\|_{F}^{2},
$$

where $\mathbf{B}^{\dagger}=\left(\mathbf{B}^{T} \mathbf{B}\right)^{-1} \mathbf{B}^{T}$ is the Moore-Penrose matrix pseudo-inverse of $\mathbf{B}$, and $\|\cdot\|_{F}$ is the Frobenius matrix norm. This measure essentially compares the variancecovariance structure between two matrices. A concordance value of one results when $\mathbf{A}^{T} \mathbf{A}=\mathbf{B}^{T} \mathbf{B}$. The concordance value is less than one when there is, on average, less variance in $\mathbf{A}$ than $\mathbf{B}$, and the concordance value is greater than one in the reverse case. Statistical characteristics of common-basis concordance will be derived in the next section. The rest of this section derives some deterministic characteristics.

Proposition 1. If the common-basis concordance conditions specified above hold, then

$$
\left\|\mathbf{A B}^{\dagger}\right\|_{F}^{2}=\sum_{i=1}^{d} \frac{\lambda_{A}(i)}{\lambda_{B}(i)}
$$

where $\lambda_{A}(i)$ and $\lambda_{B}(i)$ are the ith eigenvalues of $\mathbf{A}^{T} \mathbf{A}$ and $\mathbf{B}^{T} \mathbf{B}$ respectively.

Proof.

$$
\begin{aligned}
\left\|\mathbf{A B}^{\dagger}\right\|_{F}^{2} & =\operatorname{tr}\left(\mathbf{B}\left(\mathbf{B}^{T} \mathbf{B}\right)^{-1} \mathbf{A}^{T} \mathbf{A}\left(\mathbf{B}^{T} \mathbf{B}\right)^{-1} \mathbf{B}^{T}\right) \\
& =\operatorname{tr}\left(\mathbf{B}^{T} \mathbf{B}\left(\mathbf{B}^{T} \mathbf{B}\right)^{-1} \mathbf{A}^{T} \mathbf{A}\left(\mathbf{B}^{T} \mathbf{B}\right)^{-1}\right) \\
& =\operatorname{tr}\left(\mathbf{A}^{T} \mathbf{A}\left(\mathbf{B}^{T} \mathbf{B}\right)^{-1}\right) \\
& =\operatorname{tr}\left(\mathbf{V}^{T} \lambda_{A} \mathbf{V} \mathbf{V}^{T} \lambda_{B}^{-1} \mathbf{V}\right) \\
& =\operatorname{tr}\left(\mathbf{V} \mathbf{V}^{T} \lambda_{A} \lambda_{B}^{-1}\right) \\
& =\operatorname{tr}\left(\lambda_{A} \lambda_{B}^{-1}\right)
\end{aligned}
$$

Steps 7 and 9 follow from the cyclic permutation property of the trace. 
Corollary 1. The matrix concordance remains unchanged if both $\mathbf{A}$ and $\mathbf{B}$ are rightmultiplied by any orthonormal matrix, in particular the eigenvector matrix $\mathbf{V}$.

The corollary follows directly from $\left\|\mathbf{A V}(\mathbf{B V})^{\dagger}\right\|_{F}^{2}=\left\|\mathbf{A V V}^{T} \mathbf{B}^{\dagger}\right\|_{F}^{2}=\left\|\mathbf{A B}^{\dagger}\right\|_{F}^{2}$. Proposition 1 and Corollary 1 require that eigenvectors of the scatter matrices of $\mathbf{A}$ and $\mathbf{B}$ are the same to show that the concordance of their variance-covariance structure only depends on their eigenvalues.

The matrix concordance for matrices sharing a common scatter matrix eigenvector basis is the ratios of the eigenvalues of their scatter matrices. Applied to data-analytic statistical challenges, concordance between two data matrices can be analyzed to see if both are samples from the same distribution. Furthermore concordance allows us to estimate how well a sample from a data set captures the variance-covariance structure of the entire data set.

\section{Deriving the Ratio Distributions from the Concor- dance Statistic}

The previous section proposed a measure of concordance between two matrices with the same variance-covariance structure based on the Frobenius norm of one matrix normalized by the pseudo-inverse of the other. Furthermore, it was shown that the proposed concordance measure is preserved when each of the two matrices is rightmultiplied by the eigenvector matrix of the shared variance-covariance matrix. In this section, matrices will be assumed to be drawn at random from a specified distribution and the concordance's distribution will be derived.

In particular, it will be assumed that the data to be analyzed is drawn from some distribution with zero mean and known variance-covariance matrix $\Sigma$. A data set with $n$ samples will be denoted $\mathbf{X}_{[n]}$ indicating that the data set is made up of the set of samples from 1 to $n$. By introducing this absolute index to the samples we can easily express the first $i$ samples in $\mathbf{X}_{[n]}$ as $\mathbf{X}_{[i]}$ for $i \leq n$. Likewise, we can express all of the samples except the first $i$ as $\mathbf{X}_{[n] \backslash[i]}$. When the concordance is calculated between an entire data set and a subset it will be referred to as overlapping and when the data sets are disjoint they will be referred to as non-overlapping.

The concordance $S\left(\mathbf{X}_{[i]}, \mathbf{X}_{[n]}\right)$ normalizes the variance-covariance matrix of $\mathbf{X}_{[i]}$ by $\mathbf{X}_{[n]}$. By Corollary 1 this is equivalent to projecting the data onto the common orthonormal column basis. The resulting orthonormalized variance-covariance matrix has zero expected values for all off-diagonal elements. The diagonal elements are the ratios of the common variance estimates, which reduce to a sum of random variables centered at one with standardized dispersion.

Proposition 2. Suppose that $\mathbf{X}_{[n]}$ is sampled from an i.i.d. multivariate normal distribution. Then for sufficiently large d

$$
S\left(\mathbf{X}_{[i]}, \mathbf{X}_{[n]}\right) \dot{\sim} \mathcal{N}\left(1, \frac{2(n-i)}{d i(n+2)}\right)
$$

where $\mathcal{N}$ is a normal distribution with specified mean and variance parameters. 
Proof. The normed matrices in Equation 5 reduce to $d$ sums of $n$ squared standard normals. Furthermore $i$ of the samples are repeated in $\mathbf{X}_{[i]}$ and $\mathbf{X}_{[n]}$. Then the concordance can be expressed as:

$$
\begin{aligned}
S\left(\mathbf{X}_{[i]}, \mathbf{X}_{[n]}\right) & =\frac{1}{d} \sum_{j=1}^{d} \frac{\frac{1}{i} \sum_{k=1}^{i} \mathbf{M}[k, j]^{2}}{\frac{1}{n} \sum_{k=1}^{n} \mathbf{M}[k, j]^{2}} \\
& =\frac{1}{d} \sum_{j=1}^{d} \frac{\frac{1}{i} \sum_{k=1}^{i} \sigma_{j} Z_{k}^{2}}{\frac{1}{n} \sum_{k=1}^{n} \sigma_{j} Z_{k}^{2}} \\
& =\frac{1}{d} \sum_{j=1}^{d} \frac{\frac{1}{i} \sum_{k=1}^{i} Z_{k}^{2}}{\frac{1}{n} \sum_{k=1}^{n} Z_{k}^{2}}
\end{aligned}
$$

where $\mathbf{M}=\mathbf{X}_{[n]} \mathbf{V}$ and $\sigma_{j}$ is the $j$ th eigenvalue of $\Sigma$ and $Z_{k}$ is distributed as standard normal. Each of the $d$ terms in the summation are a ratio $\chi^{2}$ random variables. The degrees of freedom in the numerator and denominator are equal to $i$ and $n$ respectively with $i$ of the sampled random variables appearing in both the numerator and denominator. The ratio of $\chi^{2}$ distributions, where samples are repeated in the denominator, is distributed as Beta. The result follows by applying the central limit theorem to the sample mean of $d$ independent Beta distributions each of which are multiplied by the constant $n / i$.

Proposition 3. Assume the same conditions as in Proposition 2 along with the added condition that $n>>i>2$. Then

$$
S\left(\mathbf{X}_{[i]}, \mathbf{X}_{[n] \backslash[i]}\right) \dot{\sim} \mathcal{N}\left(1, \frac{2 n}{i}\right)
$$

Proof. By definition, the concordance is the same as in Equation 10 except that the summation in the denominator goes from $i+1$ to $n$. As a result we get a result similar to Equation 11 where

$$
S\left(\mathbf{X}_{[i]}, \mathbf{X}_{[n]}\right)=\frac{1}{d} \sum_{j=1}^{d} \frac{\frac{1}{i} \sum_{k=1}^{i} Z_{k}^{2}}{\frac{1}{n-i} \sum_{k=i+1}^{n} Z_{k}^{2}} .
$$

The ratio of independent $\chi^{2}$ distributions, where the numerator and denominator are normalized by their respective degrees of freedom, is $F$ with $i$ and $n-i$. The result follows by applying the central limit theorem to the sample mean of $d$ independent $F$ distributions.

Proposition 4. Suppose that $\mathbf{X}_{[n]}$ is sampled from some distribution with constant mean and variance-covariance. Let $\mathbf{M}$ be $\mathbf{X}_{[n]} \mathbf{V}$ as before. If $1 \leq j \leq d, Z$ is standard normal, and the following joint convergence holds

$$
\left[\frac{1}{n-i} \sum_{k=i+1}^{n} \mathbf{M}[k, j]^{2}, \frac{1}{i} \sum_{k=1}^{i} \mathbf{M}[k, j]^{2}\right] \rightarrow_{d}\left[1+\frac{\sigma_{j}}{\sqrt{n-i}} Z, 1+\frac{\sigma_{j}}{\sqrt{i}} Z\right] .
$$


Then the concordance is approximately distributed as Cauchy with location parameter 1 and scale parameter $\sqrt{(n-i) / i}$.

$$
S\left(\mathbf{X}_{[i]}, \mathbf{X}_{[n] \backslash[i]}\right) \dot{\sim} \text { Cauchy }\left(1, \sqrt{\frac{n-i}{i}}\right)
$$

Proof.

$$
\begin{aligned}
S\left(\mathbf{X}_{[i]}, \mathbf{X}_{[n]}\right) & =\frac{1}{d} \sum_{j=1}^{d} \frac{\frac{1}{i} \sum_{k=1}^{i} \mathbf{M}[k, j]^{2}}{\frac{1}{n-i} \sum_{k=i+1}^{n} \mathbf{M}[k, j]^{2}} \\
& \rightarrow_{d} \frac{1}{d} \sum_{j=1}^{d} \frac{\sigma_{j} Z / \sqrt{i}}{\sigma_{j} Z / \sqrt{n-i}} \\
& =\frac{1}{d} \sum_{j=1}^{d} \frac{\sqrt{n-i} Z}{\sqrt{i} Z}
\end{aligned}
$$

Each term in the summation is a ratio of two normal random variables. Equation 14 follows from the joint convergence assumption (Equation 4) and the application of the continuous mapping theorem. The ratio of two normally distributed random variables, with common location parameter, is distributed as Cauchy. The result follows by realizing that the sample mean of independent Cauchy distributions, with common location and scale parameters is Cauchy with the same location and scale parameter.

The scale and location values based on the distributional results are shown in Table 1 It may be noted that, while the Cauchy approximation relies on fewer distributional assumptions it is also less applicable. In particular its support includes the negative reals. This is a result of taking the ratio of central limit theorem approximation of two random variables, as shown in Equation 14. However, this derivation may suggest that, when the normal distribution assumptions do not hold the resulting concordance distribution is heavy-tailed.

\begin{tabular}{|c|c|c|c|}
\hline Model & Location & Scale & $\begin{array}{c}\text { Approx. Concordance } \\
\text { Distr. }\end{array}$ \\
\hline$\frac{n}{i} \operatorname{Beta}\left(\frac{i}{2}, \frac{n-i}{2}\right)$ & 1 & $\frac{2(n-i)}{i(n+2)}$ & $\mathcal{N}\left(1, \frac{2(n-i)}{\operatorname{din}}\right)$ \\
\hline$F(i, n-i)$ & $\frac{n-i}{n-i-2}$ & $\frac{2(n-i)^{2}(n-2)}{i(n-i-2)^{2}(n-i-4)}$ & $\mathcal{N}\left(1, \frac{2 n}{\operatorname{di}(n-i)}\right)$ \\
\hline Cauchy $\left(1, \sqrt{\frac{n-i}{i}}\right)$ & 1 & $\sqrt{\frac{n-i}{i}}$ & $\operatorname{Cauchy}\left(1, \sqrt{\frac{n-i}{i}}\right)$ \\
\hline
\end{tabular}

Table 1: The derived model distributions with their location and scale parameters. Note that for the $F$ and Beta model the approximate concordance assumes $n>>i>2$. 


\section{Benchmark Description, Design, and Implementation}

To assess the behavior of the relative stability measures proposed in Equation 5 this section makes use of the "Airline on-time performance" data set (RITA, 2009), which was released for the 2009 American Statistical Association (ASA) Section on Statistical Computing and Statistical Graphics biannual data exposition. The data set includes commercial flight arrival and departure information from October 1987 to April 2008 for those carriers with at least $1 \%$ of domestic U.S. flights in a given year. In total, there is information for over 120 million flights, with 29 variables related to flight time, delay time, departure airport, arrival airport, and so on. In total, the uncompressed data set is 12 gigabytes (GB) in size. Benchmarks in this section focus on the model matrix representation of the of the variables shown below in Table 2. The model matrix under consideration will use the treatment-contrast expansion of the categorical variables and has a total of 43 columns $(d=43)$.

\begin{tabular}{|c|l|c|c|}
\hline Variable Name & Description & Type & $\begin{array}{l}\text { Number of categories } \\
\text { (if applicable) }\end{array}$ \\
\hline Year & The year of flights & categorical & 22 (1987 to 2008) \\
\hline Month & The month of flights & categorical & 12 \\
\hline DayOfWeek & The day of week of flights & categorical & 7 \\
\hline DepTime & $\begin{array}{l}\text { The departure time of flights } \\
\text { (minutes after midnight) }\end{array}$ & numeric & NA \\
\hline DepDelay & $\begin{array}{l}\text { The departure delay of flights } \\
\text { (minutes) }\end{array}$ & numeric & NA \\
\hline
\end{tabular}

Table 2: Variables that will be considered for the covariance matrix stability benchmark.

The 12 GB Airline On-time data set will likely not be considered "big" to many readers. Papers such as Kane et al. (2013) have shown how the data set can be explored and analyzed on relatively modest hardware. However, in designing the benchmarks two principles were considered before sheer data size. First, the data set is publicly available. The code included in the Supplemental Material of this paper is capable of downloading the data set and running the benchmarks. Users are encouraged to engage the data themselves and perform their own analyses. Second, the data set is large enough to investigate the scatter matrix concordance properties along with the scaling behavior of the various regression techniques described in this paper. Together, the data set and the code available with this paper provide a set of accessible and reproducible benchmarks that form a basis for instruction and subsequent research.

The benchmarks presented in the next section were written in the $\mathrm{R}$ programming environment (R Core Team, 2014) and can be run on a single machine sequentially, a single machine in parallel, or on a cluster of machines using the foreach (Revolution Analytics and Weston, 2014) package, which provides a concurrent interface to a number of different parallel computing technologies for embarrassingly parallel challenges. The implementation also makes use of the iterators and itertools 
(Revolution Analytics, 2014; Weston and Wickham, 2014) packages thereby decoupling data access from retrieval and management. The most accessible and straightforward implementation approaches are used to illustrate the methodological principles of the models and their implementation. The benchmark implementation is flexible enough to be deployed to any number of different data management, communication protocol, memory, and processing configurations. It is also easily modifiable to accommodate alternative technologies and methodologies.

\section{Benchmark Results}

This section provides benchmarks exploring the convergence of the concordance to one on an increasingly larger subset of the Airline On-time Data set alongside the convergence of the slope coefficients of a Generalized Linear Model (GLM) of the same subsets. These two sets of benchmarks establish an empirical connection between regression and concordance. However, it should also be noted that since the concordance approach does not distinguish between independent and dependent variables it provides a diagnostic not only for the regression but for any regression involving the same variables. Furthermore, since the concordance calculation for a subset of the variables can be found directly from the corresponding scatter matrices a diagnostic easily be calculated for any regression involving any subset of the variables.

\subsection{Random Sampling Similarity Convergence}

The first set of benchmarks take random samples of varying sizes from 10 to 5000 from the Airline On-time data set and calculates the overlapping and non-overlapping concordance between the random subset and the entire data set. The overlapping and non-overlapping values were equal up to seven decimal places and so only the overlapping concordance is reported.

Figure 1 shows the convergence of the concordance to the value of one when concordance is calculated using both Equation 5 directly along with the trace-equivalent version in 6 . The plot shows that both versions capture the variance-covariance structure after only several thousand samples (out of a total of approximately 120 million). Furthermore, it can be seen that the trace-equivalent version is slightly smaller than the direct calculation. This is likely because the direct calculation is more sensitive to noise in the correlation terms of the scatter matrix and this may account for the overshoot when the sample size is zero and the small overshoot when the sample size is 1,000 .

Each concordance-distribution derivation reduces to the average of $d$ similarly distributed random variables. The terms summed in the concordance measure can therefore be thought of as samples, and their distribution can be examined. This distribution information is shown in Figure 2 with varying sample size. The plot again shows little difference between the direct calculation and its trace-equivalent, especially after a few thousand random samples are taken and the variance-covariance structure becomes known. The trace equivalent does appear to be slightly positively skewed when the number of samples is smaller and then appears to be essentially symmetric, like the 


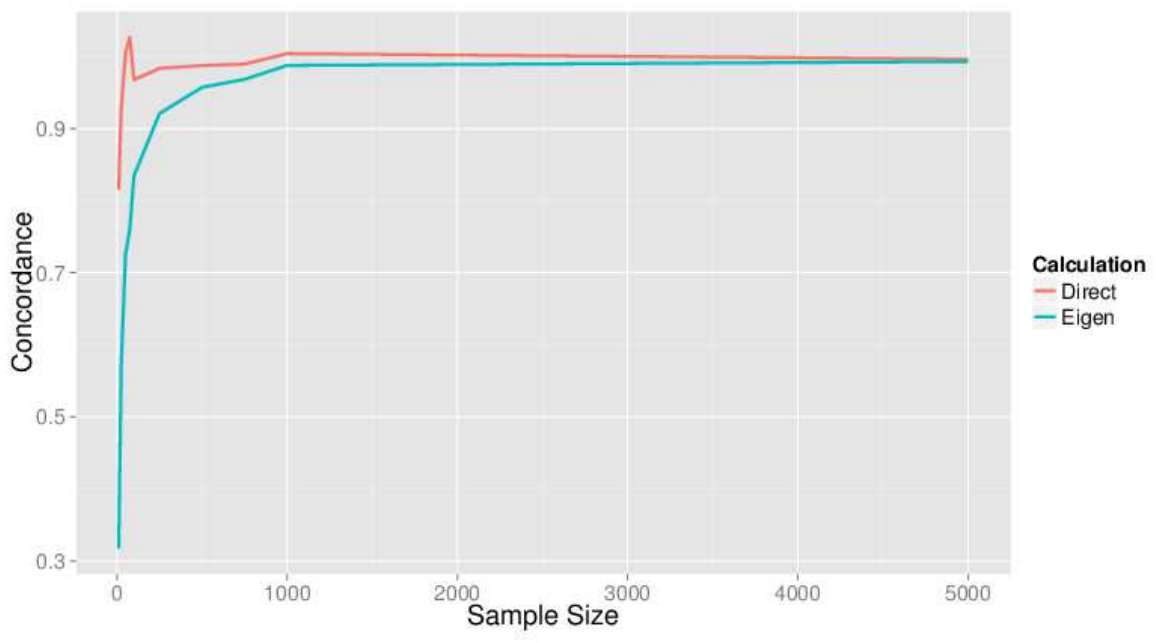

Figure 1: Convergence of concordance to one in the number of samples using random sampling.

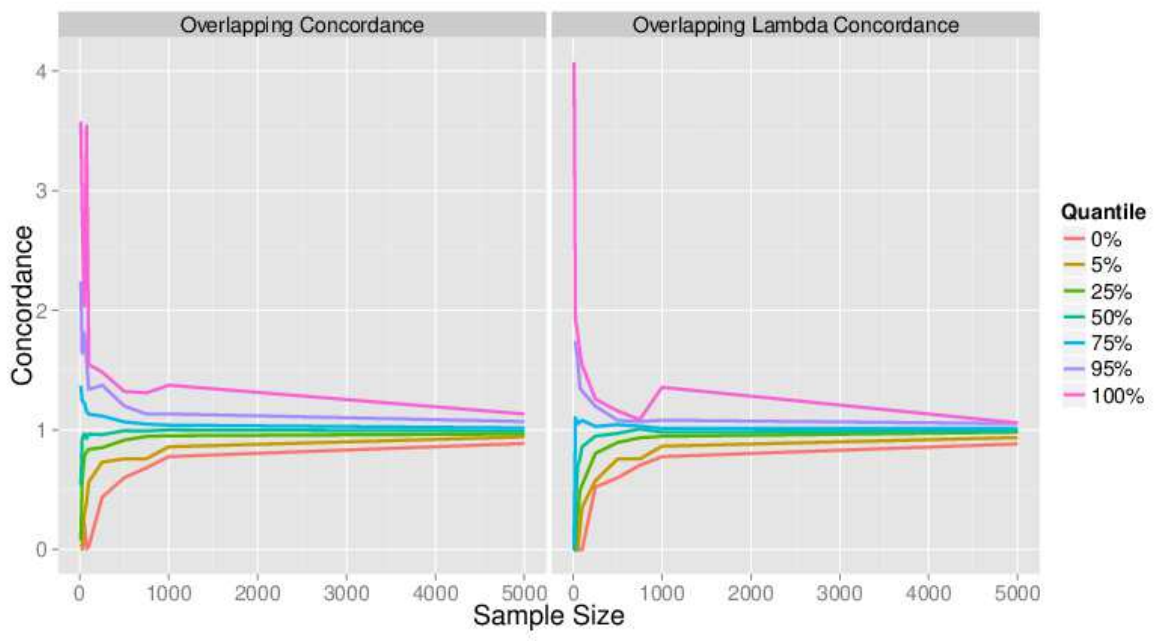

Figure 2: The distribution of overlapping and non-overlapping concordance values in the number of samples using random sampling. 


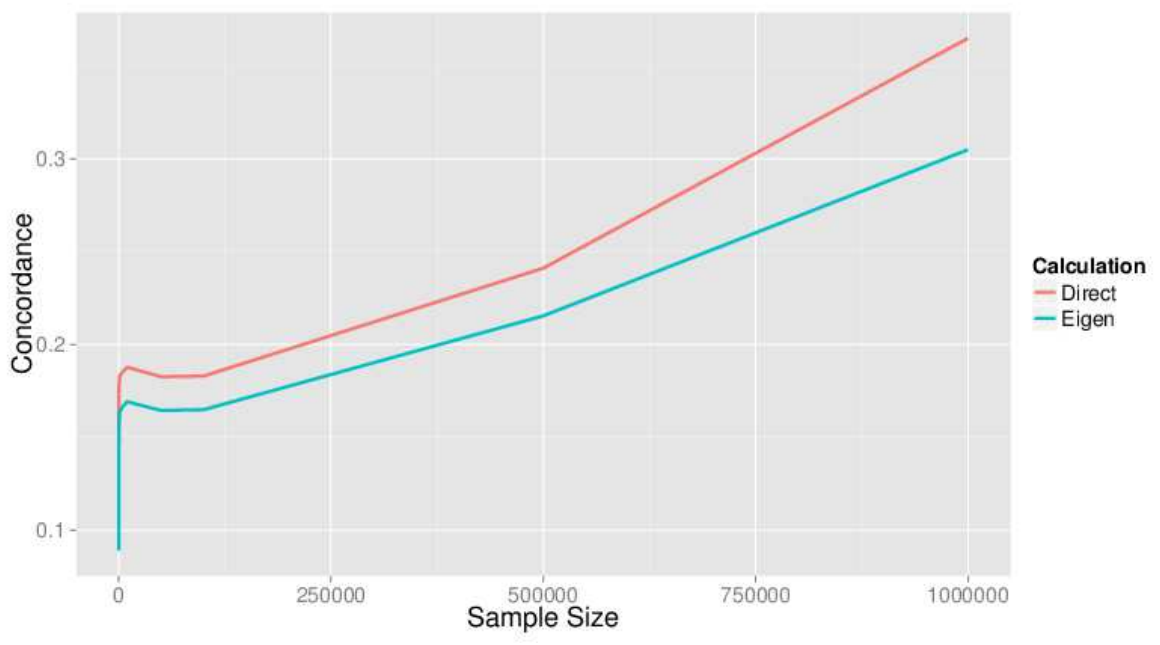

Figure 3: Convergence of concordance to one in the number of samples using nonrandom sampling.

direct calculation of the concordance. The figure also indicates that the concordance is not heavy-tailed. The $95 \%$ percentile converges relatively quickly in the number of samples.

\subsection{Non-Random Sampling Similarity Convergence}

The second set of benchmarks take contiguous samples, starting at the beginning of the Airline On-time data set, varying the size from 10 to $1,000,000$ to empirically determine the effect of not using randomized subsets when calculating concordance. Once again, the overlapping and non-overlapping concordance values were essentially identical and only the overlapping concordance is reported.

Figure 3 shows the convergence of the concordance values, once again using Equations 5 and 6 Where both versions had nearly converged to one after only a few thousand samples, non-random sampling requires millions, with the trace-equivalent concordance lagging the direct version.

Convergence in concordance is slow in the non-random case and this may be due to the fact that that the model matrix corresponding to the factor expansion does not include a sample of all of the information from the Year variable based on Figure 3 The poor convergence characteristics extend to the distribution of the concordance, as shown in Figure 3 thereby underscoring the random sampling in order to achieve representative subsets. 


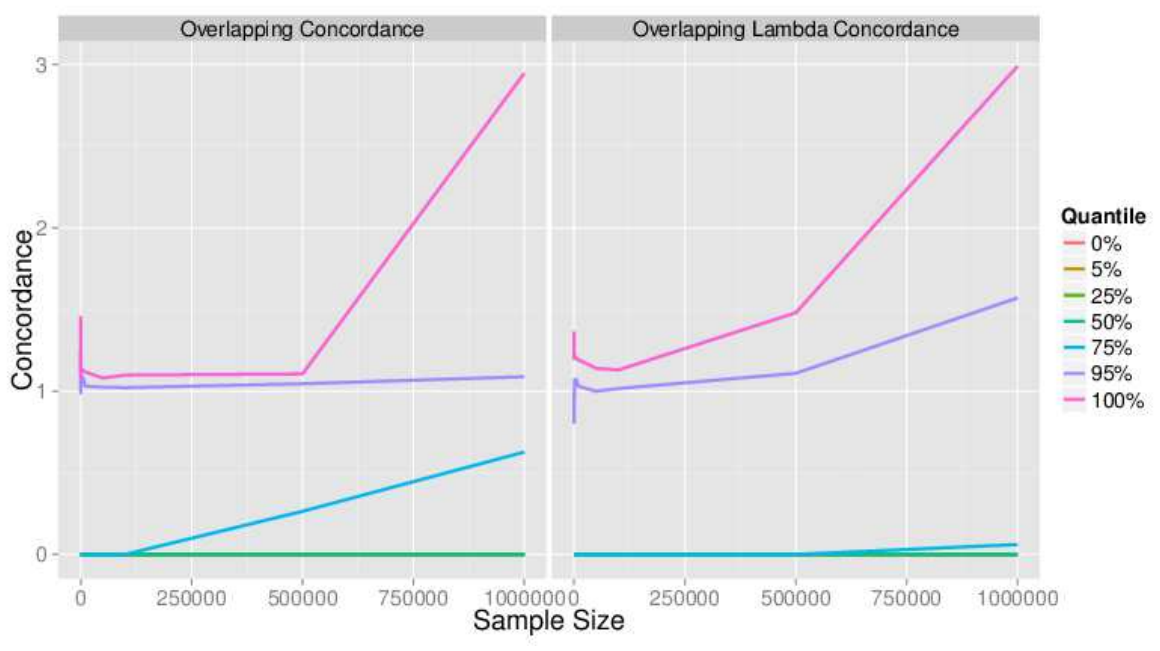

Figure 4: The distribution of overlapping and non-overlapping similarities in the number of samples using non-random sampling.

\subsection{Concordance and GLM Convergence}

The third set of benchmarks compares the concordance with the log mean square error between the slope coefficient estimates fitted using a random subset of the data and estimates fitted using the entire data set. The variable relationship under investigation is the following logistic regression:

$$
\text { Late } \sim \text { Year }+ \text { Month }+ \text { DayofWeek + DepartureTime + DepartureDelay }
$$

where a flight is "Late" if its arrival is at least 30 minutes after its scheduled arrival. The other variables are described in Table 2. For each subset size, the procedure was repeated 10 times and the average concordance and log MSE of the coefficients were recorded.

Figures 5 and 6 show the convergence behavior of the concordance to one and log MSE of the slope coefficients to zero respectively. When the number of samples is small with respect to the number of columns in the design matrix the concordance is low indicating that the variance-covariance structure is not well-represented and the log MSE of the slope coefficients is high indicating that the estimates of the coefficients is poor. These values quickly increase and decrease respectively until approximately 500 samples where both the concordance and log MSE slope decreases.

The slow convergence after 500 samples of both measurements implies that for a precise estimate of the slope coefficients, and corresponding concordance value close to one a larger subset will need to be fitted. When the subset is 1,200,000 samples (about $1 \%$ of the total data size) the concordance values is 0.9820691 and the $\log$ MSE is -2.788843 . At $12,000,000$ samples ( $10 \%$ of the data) the concordance value is 0.9822198 and the log MSE is -4.902919 . This "slow" convergence in the number 


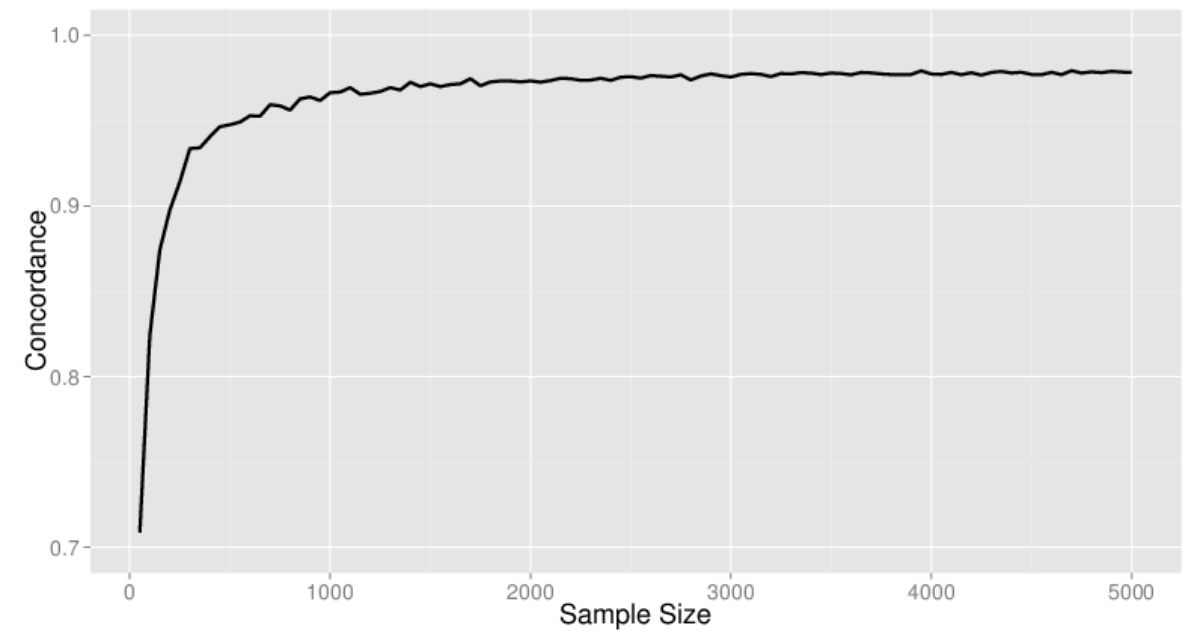

Figure 5: Convergence of concordance to one in the number of samples using random sampling.

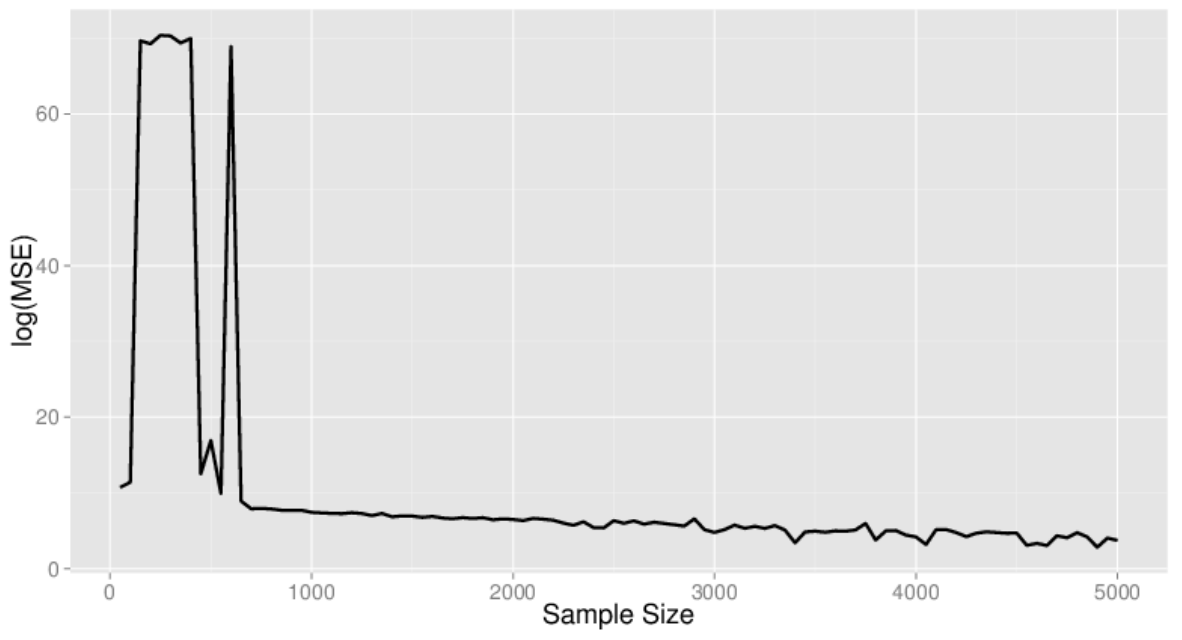

Figure 6: Convergence of log MSE between slope coefficient estimates using random subsets and the entire Airline On-time data set. 
of samples may indicative of complex relationships among the variables that require larger samples to capture.

\section{Conclusions}

Regression models depend directly on the model design matrix and its properties. This paper attempts to bridge the "small-data" and asymptotic behavior of regression models by proposing a simple measure of concordance between a design matrix and a subset of its rows that estimates how well a subset captures the variance-covariance structure of increasingly large data sets. We illustrate the use of this measure in a heuristic method for selecting row partition sizes that balance statistical and computational efficiency goals in real-world problems.

Our future work in this area will focus on data fusion. In many cases it may be desirable to combine two data sources with analogous measurements to increase the power of statistical experiments. Concordance provides a distance between the variancecovariance structure with known distributional characteristics. Tests for equivalence can then be used to rigorously assess the appropriateness of combining data sources thereby allowing practitioners to make better use of existing, potentially under-powered data.

\section{Acknowledgments}

A portion of this research is based on research sponsored by DARPA under award FA8750-12-2-0324. The U.S. Government is authorized to reproduce and distribute reprints for Governmental purposes notwithstanding any copyright notation thereon.

\section{Disclaimer}

The views and conclusions contained herein are those of the authors and should not be interpreted as necessarily representing the official policies or endorsements, either expressed or implied, of DARPA or the U.S. Government.

\section{References}

Apache Software Foundation (2014). "Apache ${ }^{\mathrm{TM}} \quad$ Hadoop®." http://hadoop.apache.org/. Accessed: 2014-01-17.

Dean J, Ghemawat S (2008). "MapReduce: Simplified Data Processing on Large Clusters." Commun. ACM, 51(1), 107-113. ISSN 0001-0782. doi:10.1145/1327452. 1327492. URL http://doi.acm.org/10.1145/1327452.1327492.

Guha S, Hafen R, Rounds J, Xia J, Li J, Xi B, Cleveland WS (2012). "Large complex data: divide and recombine (D\&R) with RHIPE." Stat, 1(1), 53-67. ISSN 20491573. doi:10.1002/sta4.7. URL http://dx.doi.org/10.1002/sta4.7. 
Kane MJ, Emerson J, Weston S (2013). "Scalable Strategies for Computing with Massive Data." Journal of Statistical Software, 55(14), 1-19. URL http://www.jstatsoft.org/v55/i14/.

Kleiner A, Talwalkar A, Sarkar P, Jordan MI (2011). "A scalable bootstrap for massive data." arXiv preprint arXiv:1112.5016.

Matloff N (2014). "Software Alchemy: Turning Complex Statistical Computations into Embarrassingly-Parallel Ones." ArXiv e-prints. 1409.5827.

R Core Team (2014). $\quad R:$ A Language and Environment for Statistical Computing. $\mathrm{R}$ Foundation for Statistical Computing, Vienna, Austria. URL http://www.R-project.org/.

Revolution Analytics (2014). iterators: Iterator construct for $R$. R package version 1.0.7, URL http://CRAN.R-project.org/package=iterators

Revolution Analytics, Weston S (2014). foreach: Foreach looping construct for $R . \quad \mathrm{R}$ package version 1.4.2, URL http://CRAN.R-project.org/package=foreach.

RITA (2009). "The Airline On-time Performance Data Set Website." Research and Innovation Technology Administration, Bureau of Transportation Statistics, URL http://stat-computing.org/dataexpo/2009/.

Weston S, Wickham H (2014). itertools: Iterator Tools. R package version 0.1-3, URL http://CRAN.R-project.org/package=itertools.

Zaharia M, Chowdhury M, Franklin MJ, Shenker S, Stoica I (2010). "Spark: Cluster Computing with Working Sets." In "Proceedings of the 2nd USENIX Conference on Hot Topics in Cloud Computing," HotCloud'10, pp. 10-10. USENIX Association, Berkeley, CA, USA. URL http://dl.acm.org/citation.cfm?id=1863103.1863113. 